\title{
Cell culture density affects the stemness gene expression of adipose tissue-derived mesenchymal stem cells
}

\author{
DAE SEONG KIM ${ }^{1}$, MYOUNG WOO LEE ${ }^{1}$, TAE-HEE LEE ${ }^{2}$,

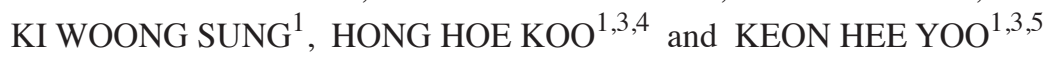

\author{
${ }^{1}$ Department of Pediatrics, Samsung Medical Center, Sungkyunkwan University School of Medicine; \\ ${ }^{2}$ Department of Laboratory of Cancer and Stem Cell Biology, Plant Engineering Institute, Sejong University; \\ ${ }^{3}$ Stem cell and Regenerative Medicine Center, Research Institute for Future Medicine, Samsung Medical Center; \\ Departments of ${ }^{4}$ Health Sciences and Technology and ${ }^{5}$ Medical Device Management and Research, \\ SAIHST, Sungkyunkwan University, Seoul, Republic of Korea
}

Received October 20,2016; Accepted November 11, 2016

DOI: $10.3892 / b r .2017 .845$

\begin{abstract}
The results of clinical trials using mesenchymal stem cells (MSCs) are controversial due to the heterogeneity of human MSCs and differences in culture conditions. In this regard, it is important to identify gene expression patterns according to culture conditions, and to determine how the cells are expanded and when they should be clinically used. In the current study, stemness gene expression was investigated in adipose tissue-derived MSCs (AT-MSCs) harvested following culture at different densities. AT-MSCs were plated at a density of 200 or 5,000 cells/ $/ \mathrm{cm}^{2}$. After 7 days of culture, stemness gene expression was examined by reverse transcription-quantitative polymerase chain reaction (RT-qPCR) analysis. The proliferation rate of AT-MSCs harvested at a low density ( $\sim 50 \%$ confluent) was higher than that of AT-MSCs harvested at a high density ( $\sim 90 \%$ confluent). Although there were differences in the expression levels of stemness gene, such as octamer-binding transcription factor 4 , nanog homeobox (Nanog), SRY-box 2, Kruppel like factor 4, v-myc avian myelocytomatosis viral oncogene homolog $(c-M y c)$, and lin-28 homolog A, in the AT-MSCs obtained from different donors, RT-qPCR analysis demonstrated differential gene expression patterns according to the cell culture density. Expression levels of stemness genes, particularly Nanog and $c-M y c$, were upregulated in AT-MSCs harvested at a low density ( $\sim 50 \%$ confluent) in comparison to AT-MSCs from the same donor harvested at a high density ( $\sim 90 \%$ confluent).
\end{abstract}

Correspondence to: Professor Keon Hee Yoo or Professor Myoung Woo Lee, Department of Pediatrics, Samsung Medical Center, Sungkyunkwan University School of Medicine, 50 Irwon-dong, Seoul 135-710, Republic of Korea

E-mail: hema2170@skku.edu

E-mail: mwlee77@hanmail.net

Key words: cell density, mesenchymal stem cell, stemness
These results imply that culture conditions, such as the cell density at harvesting, modulate the stemness gene expression and proliferation of MSCs.

\section{Introduction}

Human mesenchymal stem cells (MSCs) are promising candidates for cell-based transplantation and regenerative medicine therapeutic strategies owing to their homing ability, immunosuppressive effects $(1,2)$, capacity for self-renewal, and multilineage differentiation potential $(3,4)$. Despite these desirable features of MSCs, numerous studies and clinical trials using MSCs have yielded conflicting results. This variability may reflect differences between individual donors or differences in the culture and expansion methods used by individual investigators. In particular, culture and expansion of MSCs is a critical procedure to develop and maintain MSCs used for cell therapy. Therefore, for successful transplantation therapy, it is necessary to identify suitable methods to expand MSCs in culture that could minimize their heterogeneity.

MSCs maintain in vitro stemness and possess an extensive proliferative potential (5-7), although long-term in vitro culture and aging cause senescence and apoptosis of MSCs (8-10), in contrast to embryonic stem cells (ESCs). Previous reports demonstrated changes in gene expression, cell morphology and proliferation rate according to the cell culture density $(5,6,11-13)$, implying that this density may be a critical factor for determining the characteristics of MSCs and modulating gene expression patterns. Thus, it is important to determine how the cells are expanded and when they should be clinically used. Unfortunately, protocols and methods, including optimized culture conditions for the harvest of MSCs, have not been standardized, which has been implicated regarding the failure of clinical trials.

The current study investigated differences in stemness gene expression and the proliferation rate of adipose tissue-derived MSCs (AT-MSCs) according to the cell culture density. Although bone marrow-derived MSCs (BM-MSCs) are the most common and well-characterized type of MSCs, AT-MSCs have various advantages, as adipose tissue is abundant, easily 
accessible, collected by less invasive procedures and tends to self-replenish $(14,15)$. Differences in stemness gene expression were examined according to the donor from which AT-MSCs were isolated and the culture conditions that were used. In addition, the most appropriate method of preparing MSCs for clinical applications was evaluated.

\section{Materials and methods}

Isolation and culture of AT-MSCs. The Institutional Review Board (IRB) of Samsung Medical Center approved the current study (IRB no. 2009-09-033) and all samples were obtained with written informed consent. Adipose tissue was taken from the thigh region of three female donors during cosmetic surgery. AT-MSCs were isolated and cultured according to a previous protocol (14). Briefly, adipose tissue was washed extensively with equal volumes of HyClone Dulbecco's phosphate-buffered saline (DPBS; GE Healthcare Life Sciences Logan, UT, USA), and the extracellular matrix was digested with $0.075 \%$ collagenase A (Roche Applied Science, Penzberg, Germany) at $37^{\circ} \mathrm{C}$ for $30 \mathrm{~min}$. Enzyme activity was neutralized with low-glucose Dulbecco's modified Eagle's medium (LG-DMEM; Invitrogen-Gibco; Thermo Fisher Scientific, Inc., Waltham, MA, USA) containing 10\% fetal bovine serum (FBS; Invitrogen-Gibco) and $100 \mathrm{U} / \mathrm{ml}$ penicillin/streptomycin (PS; Invitrogen-Gibco). Samples were then centrifuged at $1,200 \mathrm{x}$ g for $10 \mathrm{~min}$. The cell pellet was washed with DPBS and filtered through a $100-\mu \mathrm{m}$ nylon mesh (cell strainer; BD Biosciences, Franklin Lakes, NJ, USA). Cells were seeded on T25 culture flasks (Nalge Nunc International, Naperville, IL, USA) at a density of $3 \times 10^{5}$ cells $/ \mathrm{cm}^{2}$ in LG-DMEM containing $10 \% \mathrm{FBS}$ and $100 \mathrm{U} / \mathrm{ml} \mathrm{PS}$. The cells were incubated in a humidified atmosphere at $37^{\circ} \mathrm{C}$ with $5 \% \mathrm{CO}_{2}$, and the medium was changed every 3-4 days until adherent fibroblast-like cells reached $\sim 70 \%$ confluency.

Culture of human induced pluripotent stem cells (iPSCs). Human iPSCs (SES8; generated from human aortic vascular smooth muscle cells) (16) were cultured on 60-mm dishes containing mitomycin C-treated mouse embryonic fibroblast (MEF) feeder cells in standard human ESC medium (ReproCell Inc., Tokyo, Japan). For passaging, human iPSCs were incubated with ESC dissociation solution (ReproCell Inc.) at $37^{\circ} \mathrm{C}$ for $3 \mathrm{~min}$. When colonies detached from the dishes, the dissociation solution was aspirated. Cell clusters were washed with ESC medium and collected in $1.5-\mathrm{ml}$ tubes. After spontaneous precipitation of pellets for $5 \mathrm{~min}$, the medium was washed out and cells were resuspended in fresh medium. Cells were then transferred to another dish with MEF and maintained in ESC medium containing $5 \mathrm{ng} / \mathrm{ml}$ basic fibroblast growth factor. MEF feeder cells were maintained in DMEM containing $10 \%$ FBS and $1 \%$ PS.

Characterization of AT-MSCs by immunophenotypic analysis. Antibodies against the human antigens CD14 [fluorescein isothiocyanate (FITC) mouse monoclonal anti-human CD14; cat. no. 555397], CD34 (FITC mouse monoclonal anti-human CD34; cat. no. 555821), CD45 (FITC mouse monoclonal anti-human CD45; cat. no. 555482), CD73 [phycoerythrin (PE) mouse monoclonal anti-human CD73; cat. no. 550257], CD90
(FITC mouse monoclonal anti-human CD90; cat. no. 555595), and CD105 (PE mouse monoclonal anti-human CD105; cat. no. 560839) were purchased from BD Biosciences. A total of $5 \times 10^{5}$ cells were resuspended in $0.2 \mathrm{ml}$ DPBS and incubated with $10 \mu \mathrm{l}$ FITC- or PE-conjugated antibodies for $30 \mathrm{~min}$ at room temperature. The fluorescence intensity of cells was evaluated by flow cytometry (FACScan; BD Biosciences) and the data were analyzed using CellQuest software version 6.0 (BD Biosciences).

\section{Differentiation of AT-MSCs}

Osteogenic differentiation. Cells were plated at $5 \times 10^{5}$ cells/well in 6-well plates in LG-DMEM containing 10\% FBS, allowed to adhere overnight, and replaced with LG-DMEM containing $10 \%$ FBS supplemented with $0.1 \mu \mathrm{M}$ dexamethasone (Sigma-Aldrich; Merck KGaA, Darmstadt, Germany), $10 \mathrm{mM} \beta$-glycerolphosphate (Sigma-Aldrich; Merck KGaA), and $100 \mu \mathrm{M}$ ascorbate-2-phosphate (Sigma-Aldrich; Merck KGaA). The medium was replaced every 3 days. After 14-21 days, osteoblast differentiation was determined by alkaline phosphatase expression.

Adipogenic differentiation. As described above, cells were cultured for 14-21 days after reaching confluence in LG-DMEM containing $10 \%$ FBS, $1 \mu \mathrm{M}$ dexamethasone, $500 \mu \mathrm{M}$ isobutyl methylxanthine (Sigma-Aldrich; Merck KGaA), $100 \mu \mathrm{M}$ indomethacin (Sigma-Aldrich; Merck KGaA), and $10 \mu \mathrm{g} / \mathrm{ml}$ insulin (Sigma-Aldrich). Adipogenic differentiation was evaluated by detecting cellular accumulation of neutral lipid vacuoles via staining with Oil Red O (Sigma-Aldrich; Merck KGaA) solution.

Chondrogenic differentiation. A total of $1 \times 10^{6}$ cells were pelleted in a $15-\mathrm{ml}$ tube by centrifugation at $300 \mathrm{x}$ g for $5 \mathrm{~min}$. Pelleted cells were cultured for 14-21 days after reaching confluence in LG-DMEM containing $1 \mathrm{X}$ insulin-transferrin-selenium (Invitrogen-Gibco), $1 \mathrm{mM}$ sodium pyruvate (Invitrogen-Gibco), $0.1 \mu \mathrm{M}$ dexamethasone, $397 \mu \mathrm{g} / \mathrm{ml}$ ascorbate-2-phosphate, and $10 \mathrm{ng} / \mathrm{ml}$ transforming growth factor- $\beta 1$ (R\&D Systems, Inc., Minneapolis, MN, USA). Chondrogenic induction was evaluated at $80 \%$ confluence by detecting extracellular accumulation of chondrocyte matrix by Toluidine blue (Sigma-Aldrich; Merck KGaA) staining.

Phase-contrast microscopy and cell counting. Viable third-passage AT-MSCs were used throughout the present study. AT-MSCs plated at an initial density of 200 or $5,000 \mathrm{cells} / \mathrm{cm}^{2}$ were observed on day 7 of culture using a phase-contrast microscope (Olympus CK40; Olympus America Inc, Melville, NY, USA). Confluency was estimated by phase-contrast microscopy based on the occupied surface of the tissue culture flask. The total cell number was determined using a hemocytometer (Marienfeld-Superior; Lauda-Königshofen, Germany).

Reverse transcription-quantitative polymerase chain reaction (RT-qPCR) analysis. Cultured cells were collected by treatment with $0.05 \%$ trypsin-EDTA (Invitrogen-Gibco; Thermo Fisher Scientific, Inc.). Total cellular RNA was extracted from pelleted cells and purified using a Qiagen RNeasy Mini kit (Qiagen, Inc., Valencia, CA, USA) according to the manufacturer's 
Table I. Primer sequences used for reverse transcription-quantitative polymerase chain reaction.

\begin{tabular}{llc}
\hline Target & \multicolumn{1}{c}{ Primer sequence } & Product size, bp \\
\hline Oct4 & F: 5'-CACACTGCAGCAGATCAGCC-3' & 328 \\
Rox2 & R: 5'-ATGATCGATTCAGTTTGAATGCATGGGAGAGCC-3' & \\
& F: 5'-TTGTTCGATCCCAACTTTCC-3' & \\
Nanog & R: 5'-ACATGGATTCTCGGCAGACT-3' & 198 \\
& F: 5'-GTGGAGCAACCAGACCTGGA-3' & 343 \\
Klf4 & R: 5'-ATCGATTCACACGTCTTCAGGTTGCATGTT-3' & \\
& F: 5'-TATGACCCACACTGCCAGAA-3' & 197 \\
c-Myc & R: 5'-ATCCAGTCACAGACCCCATC-3' & \\
& F: 5'-TTCGGGTAGTGGAAAACCAG-3' & 202 \\
Lin28 & R: 5'-AGCAGCTCGAATTTCTTC-3' & 200 \\
GAPDH & F: 5'-AATGCAAGTGAGGGTTCTGG-3' & \\
& R: 5'-CTTGGCTCCATGAATCTGGT-3' & 87
\end{tabular}

Oct4, octamer-binding transcription factor 4; Nanog, nanog homeobox; Sox2, SRY-box 2; Klf4, Kruppel-like factor 4; c-Myc, v-myc avian myelocytomatosis viral oncogene homolog; GAPDH, glyceraldehyde-3-phosphate dehydrogenase; F, forward; R, reverse.

protocol. RNA quality was determined by denaturing $1 \%$ agarose gel electrophoresis ( $120 \mathrm{~V}$ for $20 \mathrm{~min}$ ), the OD 260/280 ratio, and analysis on an Agilent 2100 Bioanalyzer (Agilent Technologies, Inc., Palo Alto, CA, USA). cDNA was produced using the Superscript RT-PCR system (Invitrogen $\mathrm{GmbH}$, Karlsruhe, Germany) according to the manufacturer's recommendations for oligo (dT)20-primed cDNA synthesis. RT-qPCR was performed in 384-well microtiter plates using a gene-specific TaqMan probe and primer sets (Applied Biosystems Assays-on-Demand; Thermo Fisher Scientific, Inc.) and an ABI PRISM 7900HT Sequence Detection System (Applied Biosystems; Thermo Fisher Scientific, Inc.). Template cDNA was added to the reaction mixture, and amplifications were initiated with a template denaturation step of $95^{\circ} \mathrm{C}$ for $10 \mathrm{~min}$, followed by 40 cycles of $95^{\circ} \mathrm{C}$ for $15 \mathrm{sec}$ and $60^{\circ} \mathrm{C}$ for $1 \mathrm{~min}$. All samples were amplified in triplicate. Data were analyzed using Sequence Detector software version 2.2.1 (Applied Biosystems; Thermo Fisher Scientific, Inc.). cDNAs were amplified using the primers presented in Table I.

Statistical analysis. Data are expressed as means \pm standard deviation. Statistical significance was determined using Student's t-test and $\mathrm{P}<0.05$ was considered to indicate a statistically significant difference.

\section{Results}

Characteristics of AT-MSCs. AT-MSCs were obtained from the mononuclear cell fraction of adipose tissue samples derived from three donors. Cells were selected based on plastic adherence to ensure the removal of any contaminating hematopoietic cells. The surface antigen profiles of these cells were analyzed by fluorescence-activated cell sorting. AT MSCs expressed characteristic stem cell associated surface markers (CD73, CD90 and CD105) and did not express hematopoietic antigens (CD14, CD34 and CD45; Fig. 1A). AT MSCs expanded easily in vitro and exhibited a fibroblast like morphology (Fig. 1B). Furthermore, expanded cells maintained the potential to differentiate into osteoblasts, adipocytes and chondrocytes (Fig. 1B) indicating that all three populations comprised of MSCs.

Proliferation rate of AT-MSCs differs according to the cell density at harvesting. AT-MSCs derived from three different donors were plated at a density of 200 or 5,000 cells $/ \mathrm{cm}^{2}$ and incubated for 7 days. Cultures plated at a density of 200 cells $/ \mathrm{cm}^{2}$ were $~ 50 \%$ confluent after 7 days, and most cells were thin and spindle-shaped (Fig. 2A). Cultures plated at a density of 5,000 cells $/ \mathrm{cm}^{2}$ were $~ 90 \%$ confluent after 7 days, and most cells had extensive cell-to-cell contacts (Fig. 2A). The number of times that cells divided in each culture condition was similar among the three donor samples (D1-D3; Fig. 2B). However, AT-MSCs from each of the three donors exhibited differences in their proliferation rate between the two culture conditions. After 7 days, AT-MSCs from D1, D2 and D3 plated at a density of 200 cells $/ \mathrm{cm}^{2}$ had divided $2.46 \pm 0.42,3.03 \pm 0.48$, and 3.18 \pm 0.45 times, respectively, whereas AT-MSCs from D1, D2 and D3 plated at a density of 5,000 cells $/ \mathrm{cm}^{2}$ had divided $1.08 \pm 0.32,1.19 \pm 0.40$, and $1.30 \pm 0.38$ times, respectively.

Stemness gene expression levels are higher in AT-MSCs harvested at a low density than in AT-MSCs harvested at a high density. RT-qPCR analysis was performed to identify stemness gene expression in AT-MSCs plated at different densities. The expression levels of stemness genes, particularly octamer-binding transcription factor $4(\mathrm{Oct} 4)$, nanog homeobox (Nanog), SRY-box 2 (Sox2), Kruppel like factor 4 (Klf4), v-myc avian myelocytomatosis viral 


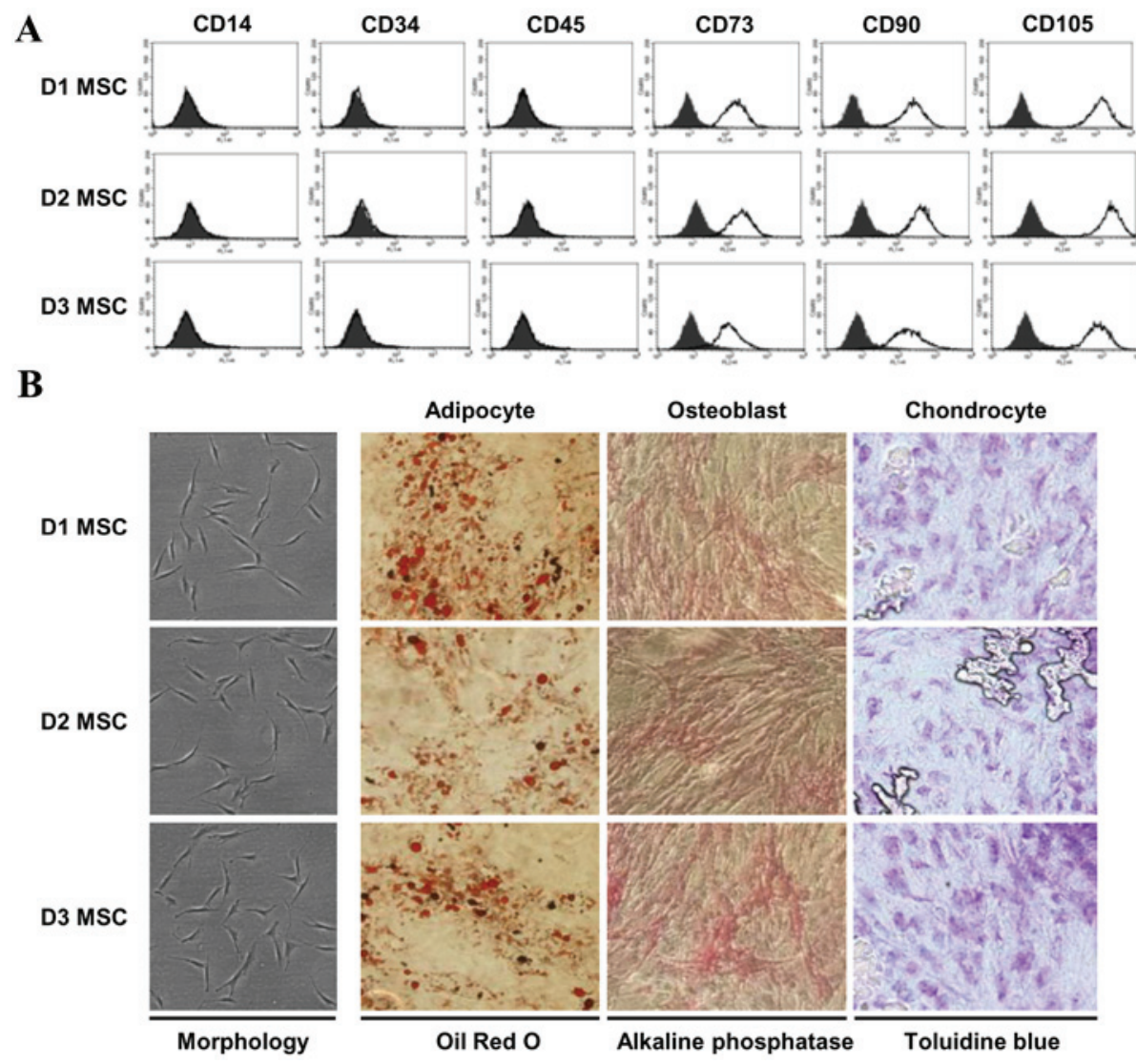

Figure 1. Characteristics of AT-MSCs obtained from three different donors. (A) Immunophenotypes of AT-MSCs obtained from three donors (D1, D2 and D3) were analyzed by flow cytometry. The expression of surface antigens is plotted against appropriate IgG isotype controls (black histogram). AT-MSCs used for analyses were positive for CD73, CD90 and CD105, and negative for CD14, CD34 and CD45 (clear histogram). (B) Morphological appearance and differentiation of AT-MSCs from three donors. MSCs observed using an inverted microscope and were spindle-shaped or exhibited a fibroblastic morphology. Cells were incubated for 14-21 days in the presence of specific differentiation agents for osteoblasts, chondrocytes and adipocytes. Alkaline phosphatase staining demonstrates mineralization of the extracellular matrix. Toluidine blue staining demonstrates the deposition of proteoglycans and lacunae. Differentiation into the adipocyte lineage was demonstrated by Oil Red O staining (magnification, x100). CD14, monocyte antigen; CD34 hematopoietic stem cell antigen; CD45, leukocyte common antigen; CD73, SH3/4; CD90, thy-1; CD105, endoglin; AT-MSC, adipose tissue-derived mesenchymal stem cell.
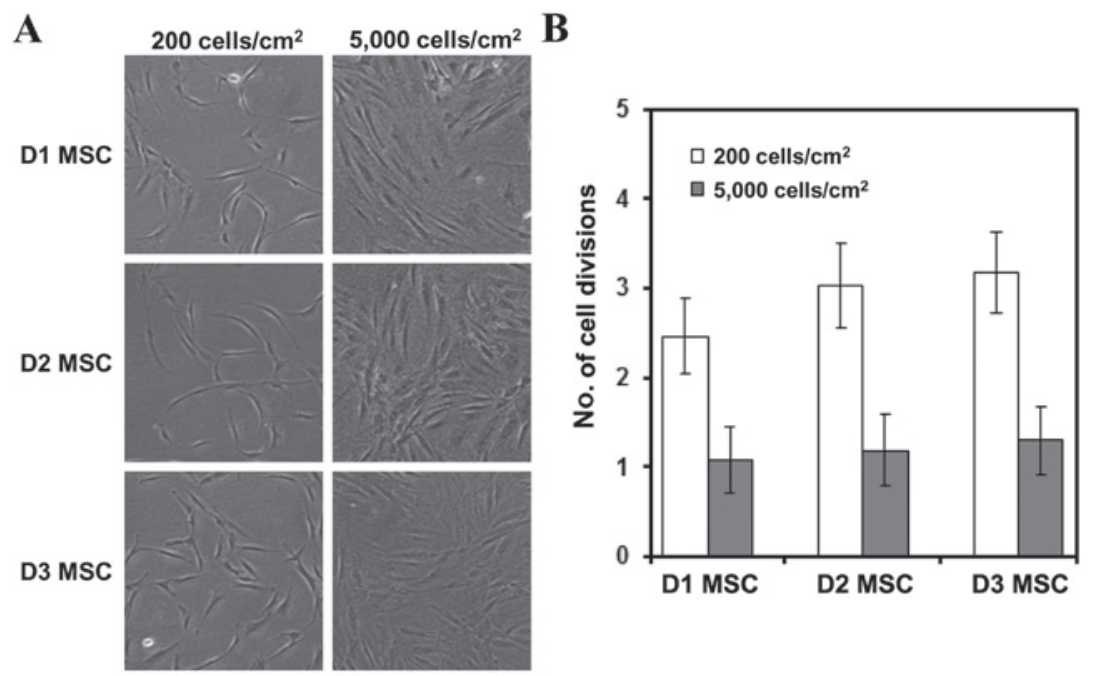

Figure 2. Phase-contrast micrographs and the number of AT-MSC divisions according to the cell culture density. (A) Morphological appearance of AT-MSCs from three donors (D1, D2 and D3) 7 days after they were plated at a density of 200 or 5,000 cells $/ \mathrm{cm}^{2}$. All cells were spindle-shaped or exhibited a fibroblastic morphology. (B) Number of cell divisions at the time of harvest of MSCs cultured under different conditions. Data are the mean \pm standard deviation of three separate experiments. AT-MSC, adipose tissue-derived mesenchymal stem cell.

oncogene homolog $(c-M y c)$, and lin-28 homolog A $(\operatorname{Lin} 28)$, were measured in AT-MSCs that were obtained from three donors, plated at a density of 200 or $5,000 \mathrm{cells} / \mathrm{cm}^{2}$, and cultured for 7 days. Human iPSCs (SES8) served as control 

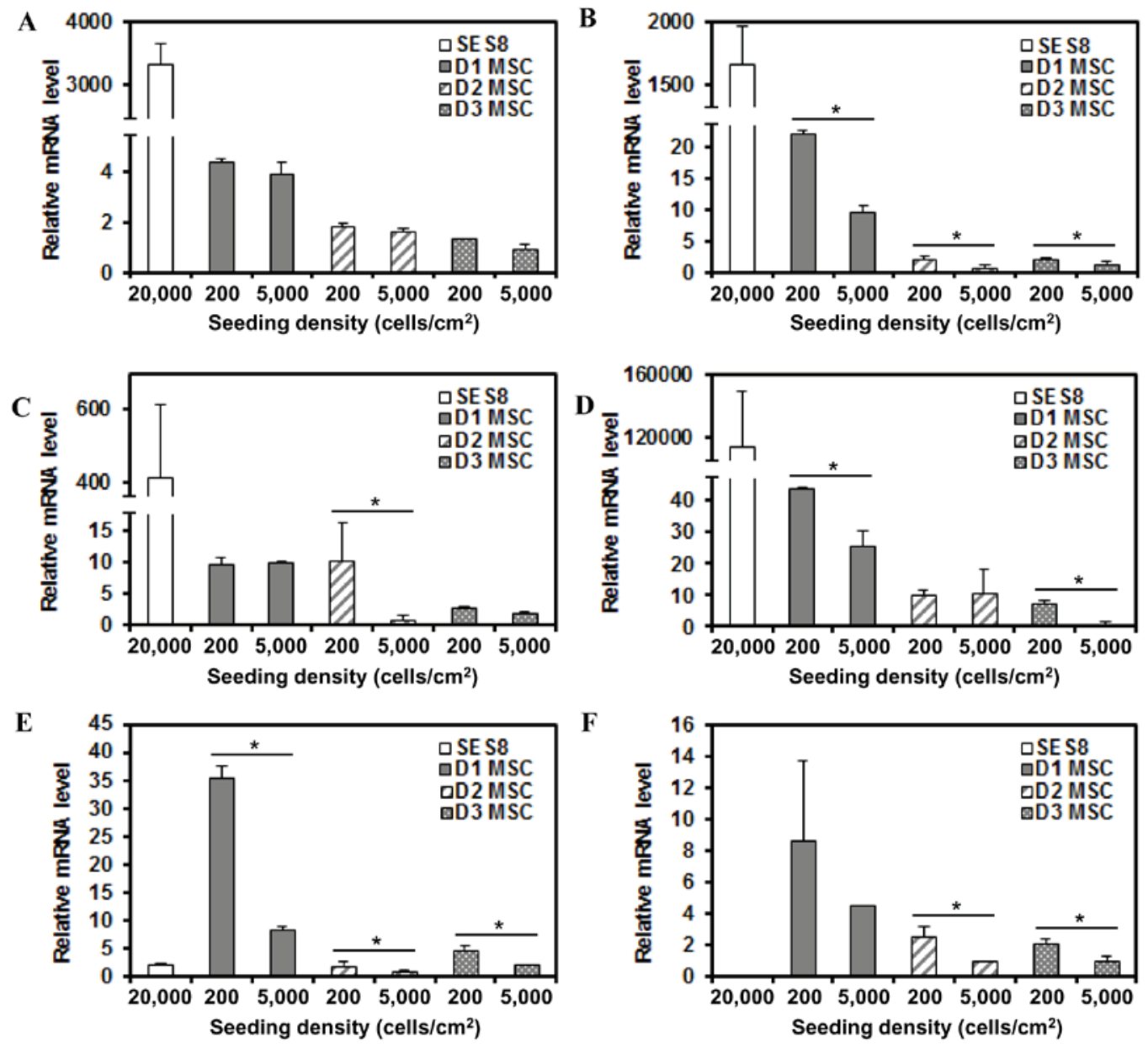

Figure 3. Reverse transcription-quantitative polymerase chain reaction (RT-qPCR) analysis of stemness genes in AT-MSCs from different donors and cultured at different densities. Stemness gene expression in AT-MSCs from three donors (D1, D2, and D3) harvested 7 days after they were plated at different densities was examined by RT-qPCR. (A-F) Quantitative gene expression data of (A) Oct4, (B) Nanog, (C) Sox2, (D) Lin28, (E) c-Myc and (F) Klf4 normalized against that of glyceraldehyde-3-phosphate dehydrogenase. mRNA levels are shown relative to the sample in which expression was lowest. ${ }^{*} \mathrm{P}<0.05$. RT-qPCR, reverse transcription-quantitative polymerase chain reaction; AT-MSCs, adipose tissue-derived mesenchymal stem cell; Oct4, octamer-binding transcription factor 4; Nanog, nanog homeobox; Sox2, SRY-box 2; Klf4, Kruppel-like factor 4; c-Myc, v-myc avian myelocytomatosis viral oncogene homolog.

cells that express stemness markers. Expression levels of Oct4, Nanog, Sox2 and Lin28 were high in SES8 cells, while expression levels of Klf4 and $c-M y c$ were high in the AT-MSCs (Fig. 3). Although there were differences in gene expression levels among AT-MSCs obtained from the different donors, RT-qPCR analysis demonstrated differential gene expression patterns according to the cell culture density. Expression levels of stemness genes, particularly Nanog and $c-M y c$, were upregulated in AT-MSCs harvested at a low density ( $\sim 50 \%$ confluent) in comparison to AT-MSCs from the same donor harvested at a high density ( $\sim 90 \%$ confluent). Expression of Oct4 did not significantly change between MSCs harvested from the different culture conditions. Among the three donors, stemness gene expression was highest in the AT-MSCs from D1.

\section{Discussion}

To the best of our knowledge, this is the first study to evaluate the stemness gene expression patterns of AT-MSCs harvested at different densities. Although MSCs were derived from different donors, cells harvested at a low density ( $\sim 50 \%$ confluent $)$ exhibited a higher proliferation rate and stemness gene expression than cells harvested at a high density ( $\sim 90 \%$ confluent). These results imply that the cell culture density is a critical factor for modulating the specific gene expression patterns of heterogeneous MSCs.

In the current study, iPSCs served as control cells, which express stemness markers, such as Oct4, Sox2, Nanog, c-Myc, Klf4 and Lin28. The recent landmark discovery that mouse and human somatic cells are reprogrammed into a ground state of pluripotency by ectopic expression of only a few defined transcription factors offers a novel, fascinating means to obtain patient-specific pluripotent cells, without the technical and ethical limitations of somatic cell nuclear transfer (17-19). However, the therapeutic value of reprogramming remains largely unknown due to the problems of teratoma formation (20) and the inadequate long-term stability of iPSC-derived tissues $(19,20)$. Therefore, there are significant problems that impede the adaptation of iPSCs for clinical use (21). Conversely, human MSCs pose no risk of teratoma formation and are not ethically or politically controversial. Additionally, MSCs exhibit great multipotency and pose no risk of immune rejection by the host (22-24). 
Previous studies indicate that low initial plating densities result in faster expansion, leading to higher MSC yield (5-7). MSCs contain a subpopulation of rapidly self-replicating cells $(5-7,25)$ that may have a great capacity to generate single-cell-derived clones through rapid proliferation. For this reason, cells plated at lower densities may have a higher doubling number per passage, while growth at higher densities would become constrained by density-dependent growth inhibition. The observed difference in proliferation rates between MSCs harvested at low and high densities indicates that the growth patterns of MSC cultures depend on the cell culture density. At a low density, MSCs dispersed evenly across the plate and, over time, rapidly grew to fill the available space. As they progressed toward full confluency, the proliferation of the MSCs slowed due to cell-to-cell contact, which was reflected in the reduced expression levels of stemness genes.

Previous studies have demonstrated that the stemness of MSCs, as characterized by their high proliferative potential and self-renewal capacity, is important for biomedical applications $(5-7,11,25,26)$. Another study demonstrated that Oct4/Sox2/Nanog overexpression improves the proliferation and differentiation of AT-MSCs $(27,28)$. The stemness of MSCs following their homing is required for tissue repair. Stem cell maintenance in adult organisms is essential for tissue homeostasis, the initiation of native tissue regeneration and responses to injury (29). Therefore, the identification of regulatory factors that maintain the stemness properties of MSCs is important to obtain appropriate MSCs adopted for therapeutic applications. The current results demonstrate that MSCs maintain stemness, an essential characteristic of stem cells, during explant culture. As shown in the present study, MSCs express markers characterized in ESCs, such as Oct4, Sox2, Nanog, Klf4, c-Myc and Lin28. Furthermore, Klf4 and $c-M y c$ expression levels were greater in AT-MSCs than in iPSCs. Oct4, Sox2 and Klf4, which share many target genes in ESCs, are critical components of the pluripotency circuit to maintain the self-renewal capacity of undifferentiated ESCs. According to previous studies, Oct4, Sox 2 , Lin 28 and Nanog regulate the proliferative capacity, colony formation and lineage differentiation potencies of MSCs (27,28,30-34). As a key factor in reprogramming, Klf4 functions as a transcriptional activator and repressor to regulate the proliferation and differentiation of different cell types (35). $c$-Myc induces DNA replication and cell cycle progression, and modulates the ESC epigenome, promoting the opening of chromatin to allow the access of pluripotent factors $(36,37)$. In the current study, gene expression patterns of AT-MSCs derived from three donors were investigated. Although each MSC exhibits heterogeneous characteristics with regards to their morphology, proliferation rate and secreted factors $(5-7,11,12,38)$, the expression levels of stemness genes were higher in AT-MSCs harvested at a low density than in AT-MSCs harvested at a high density. These results are consistent with our previous study demonstrating that Oct4 and Nanog gene expression is higher in BM-MSCs harvested at a low density than in BM-MSCs harvested at a high density (13), indicating that the cell culture density predominantly modulates the stemness gene expression patterns of MSCs.
In conclusion, the current study demonstrates the possibility of modulating MSC gene expression patterns by manipulating the cell density, as well as the feasibility of designing a strategy to prepare suitable MSCs using specific culture conditions, despite the heterogeneity of these cells.

\section{Acknowledgements}

The present study was supported by the Basic Science Research Program through the National Research Foundation of Korea funded by the Ministry of Science, ICT and Future Planning (grant no. 2014R1A1A1008388) and by the Ministry of Education (grant no. 2012R1A1A2041534).

\section{References}

1. Tian Y, Deng YB, Huang YJ and Wang Y: Bone marrow-derived mesenchymal stem cells decrease acute graft-versus-host disease after allogeneic hematopoietic stem cells transplantation. Immunol Invest 37: 29-42, 2008

2. Keyser KA, Beagles KE and Kiem HP: Comparison of mesenchymal stem cells from different tissues to suppress T-cell activation. Cell Transplant 16: 555-562, 2007.

3. Pittenger MF, Mackay AM, Beck SC, Jaiswal RK, Douglas R, Mosca JD, Moorman MA, Simonetti DW, Craig S and Marshak DR: Multilineage potential of adult human mesenchymal stem cells. Science 284: 143-147, 1999.

4. Sasaki M, Abe R, Fujita Y, Ando S, Inokuma D and Shimizu H: Mesenchymal stem cells are recruited into wounded skin and contribute to wound repair by transdifferentiation into multiple skin cell type. J Immunol 180: 2581-2587, 2008.

5. Colter DC, Class R, DiGirolamo CM and Prockop DJ: Rapid expansion of recycling stem cells in cultures of plastic-adherent cells from human bone marrow. Proc Natl Acad Sci USA 97: 3213-3218, 2000

6. Sekiya I, Larson BL, Smith JR, Pochampally R, Cui JG and Prockop DJ: Expansion of human adult stem cells from bone marrow stroma: Conditions that maximize the yields of early progenitors and evaluate their quality. Stem Cells 20: 530-541, 2002.

7. Lee RH, Hsu SC, Munoz J, Jung JS, Lee NR, Pochampally R and Prockop DJ: A subset of human rapidly self-renewing marrow stromal cells preferentially engraft in mice. Blood 107: 2153-2161, 2006.

8. Estrada JC, Torres Y, Benguría A, Dopazo A, Roche E, Carrera-Quintanar L, Pérez RA, Enríquez JA, Torres R, Ramírez JC, et al: Human mesenchymal stem cell-replicative senescence and oxidative stress are closely linked to aneuploidy. Cell Death Dis 4: e691, 2013.

9. Wagner W, Horn P, Castoldi M, Diehlmann A, Bork S, Saffrich R, Benes V, Blake J, Pfister S, Eckstein V, et al: Replicative senescence of mesenchymal stem cells: A continuous and organized process. PLoS One 3: e2213, 2008.

10. Schellenberg A, Lin Q, Schüler H, Koch CM, Joussen S, Denecke B, Walenda G, Pallua N, Suschek CV, Zenke M, et al: Replicative senescence of mesenchymal stem cells causes DNA-methylation changes which correlate with repressive histone marks. Aging (Albany NY) 3: 873-888, 2011.

11. Lee RH, Seo MJ, Pulin AA, Gregory CA, Ylostalo J and Prockop DJ: The CD34-like protein PODXL and alpha6-integrin (CD49f) identify early progenitor MSCs with increased clonogenicity and migration to infarcted heart in mice. Blood 113: 816-826, 2009.

12. Kim DS, Lee MW, Yoo KH, Lee TH, Kim HJ, Jang IK, Chun YH, Kim HJ,Park SJ, Lee SH, et al: Gene expression profiles of human adipose tissue-derived mesenchymal stem cells are modified by cell culture density. PLoS One 9: e83363, 2014

13. Lee MW, Kim DS, Yoo KH, Kim HR, Jang IK, Lee JH, Kim SY, Son MH, Lee SH, Jung HL, et al: Human bone marrow-derived mesenchymal stem cell gene expression patterns vary with culture conditions. Blood Res 48: 107-114, 2013.

14. Zuk PA, Zhu M, Mizuno H, Huang J, Futrell JW, Katz AJ, Benhaim P, Lorenz HP and Hedrick MH: Multilineage cells from human adipose tissue: Implications for cell-based therapies. Tissue Eng 7: 211-228, 2001. 
15. Jurgens WJ, Oedayrajsingh-Varma MJ, Helder MN Zandiehdoulabi B, Schouten TE, Kuik DJ, Ritt MJ and van Milligen FJ: Effect of tissue-harvesting site on yield of stem cells derived from adipose tissue: Implications for cell-based therapies. Cell Tissue Res 332: 415-426, 2008.

16. Lee TH, Song SH, Kim KL, Yi JY, Shin GH, Kim JY, Kim J, Han YM, Lee SH, Lee SH, et al: Functional recapitulation of smooth muscle cells via induced pluripotent stem cells from human aortic smooth muscle cells. Circ Res 106: 120-128, 2010.

17. Martinez-Fernandez A, Nelson TJ, Yamada S, Reyes S Alekseev AE, Perez-Terzic C, Ikeda Y and Terzic A: iPS programmed without c-MYC yield proficient cardiogenesis for functional heart chimerism. Circ Res 105: 648-656, 2009.

18. Mauritz C, Schwanke K, Reppel M, Neef S, Katsirntaki K, Maier LS, Nguemo F, Menke S, Haustein M, Hescheler J, et al: Generation of functional murine cardiac myocytes from induced pluripotent stem cells. Circulation 118: 507-517, 2008.

19. Takahashi K and Yamanaka S: Induction of pluripotent stem cells from mouse embryonic and adult fibroblast cultures by defined factors. Cell 126: 663-676, 2006.

20. Zhang F, Citra F and Wang DA: Prospects of induced pluripotent stem cell technology in regenerative medicine. Tissue Eng Part B Rev 17: 115-124, 2011.

21. Liu YH, Karra R and Wu SM: Cardiovascular stem cells in regenerative medicine: Ready for prime time? Drug Discov Today Ther Strateg 5: 201-207, 2008.

22. Uccelli A, Moretta L and Pistoia V: Mesenchymal stem cells in health and disease. Nat Rev Immunol 8: 726-736, 2008

23. Murphy MB, Moncivais K and Caplan AI: Mesenchymal stem cells: Environmentally responsive therapeutics for regenerative medicine. Exp Mol Med 45: e54, 2013.

24. Melief SM, Zwaginga JJ, Fibbe WE and Roelofs H: Adipose tissue-derived multipotent stromal cells have a higher immunomodulatory capacity than their bone marrow-derived counterparts. Stem Cells Transl Med 2: 455-463, 2013.

25. Smith JR, Pochampally R, Perry A, Hsu SC and Prockop DJ: Isolation of a highly clonogenic and multipotential subfraction of adult stem cells from bone marrow stroma. Stem Cells 22: 823-831, 2004

26. Pricola KL, Kuhn NZ, Haleem-Smith H, Song Y and Tuan RS: Interleukin- 6 maintains bone marrow-derived mesenchymal stem cell stemness by an ERK1/2-dependent mechanism. J Cell Biochem 108: 577-588, 2009.
27. Han SM, Han SH, Coh YR, Jang G, Chan Ra J, Kang SK, Lee HW and Youn HY: Enhanced proliferation and differentiation of Oct4- and Sox2-overexpressing human adipose tissue mesenchymal stem cells. Exp Mol Med 46: e101, 2014

28. Go MJ, Takenaka C and Ohgushi H: Forced expression of Sox2 or Nanog in human bone marrow derived mesenchymal stem cells maintains their expansion and differentiation capabilities. Exp Cell Res 314: 1147-1154, 2008

29. Alison MR and Islam S: Attributes of adult stem cells. J Pathol 217: 144-160, 2009.

30. Iovino $\mathrm{N}$ and Cavalli G: Rolling ES cells down the Waddington landscape with Oct4 and Sox2. Cell 145: 815-817, 2011.

31. Liu TM, Wu YN, Guo XM, Hui JH, Lee EH and Lim B: Effects of ectopic Nanog and Oct4 overexpression on mesenchymal stem cells. Stem Cells Dev 18: 1013-1022, 2009.

32. Tondreau T, Meuleman N, Delforge A, Dejeneffe M, Leroy R, Massy M, Mortier C, Bron D and Lagneaux L: Mesenchymal stem cells derived from CD133-positive cells in mobilized peripheral blood and cord blood: Proliferation, Oct 4 expression, and plasticity. Stem Cells 23: 1105-1112, 2005.

33. Greco SJ, Liu K and Rameshwar P: Functional similarities among genes regulated by OCT4 in human mesenchymal and embryonic stem cells. Stem Cells 25: 3143-3154, 2007.

34. Tsai CC, Su PF, Huang YF, Yew TL and Hung SC: Oct4 and Nanog directly regulate Dnmt1 to maintain self-renewal and undifferentiated state in mesenchymal stem cells. Mol Cell 47: $169-182,2012$.

35. Evans PM, Zhang W, Chen X, Yang J, Bhakat KK and Liu C: Kruppel-like factor 4 is acetylated by p300 and regulates gene transcription via modulation of histone acetylation. J Biol Chem 282: 33994-34002, 2007.

36. Scheper W and Copray S: The molecular mechanism of induced pluripotency: A two-stage switch. Stem Cell Rev 5: 204-223, 2009.

37. Varlakhanova NV, Cotterman RF, deVries WN, Morgan J, Donahue LR, Murray S, Knowles BB and Knoepfler PS: myc maintains embryonic stem cell pluripotency and self-renewal. Differentiation 80: 9-19, 2010.

38. Kern S, Eichler H, Stoeve J, Klüter H and Bieback K: Comparative analysis of mesenchymal stem cells from bone marrow, umbilical cord blood, or adipose tissue. Stem Cells 24: 1294-1301, 2006. 